\title{
Biodiversity and biotechnological applications of novel plant growth promoting methylotrophs
}

\section{Editorial}

Methylotrophic bacterial community is a very important group of bacteria utilizing reduced carbon compounds. Methylotrophic bacteria are well enough to survive in all types of environmental conditions including acidic/alkaline habitats, ${ }^{1-3}$ hyper saline, ${ }^{4,5}$ drought $^{6-8}$ low temperature ${ }^{9-11}$ and high temperature. ${ }^{6,12,13}$ The methylotrophic microbes help plant for adaptation under diverse unfavourable environmental conditions. The pink pigmented facultative methylotrophic (PPFMs) bacteria is abundantly reported as plant microbiomes (epiphytes, endophytes, rhizospheric). ${ }^{11,13,14}$ The methylotrophic microbes could be promote the plant growth and soil health for sustainable agriculture directly by N2-fixation fixation; $\mathrm{P}, \mathrm{K}$ and $\mathrm{Zn}$ solubilization; production of Fe-chelating compounds; production of PGP hormones such gibberellic acids, auxin and cytokinin and ACC deaminase activities ${ }^{6,11,13,15}$ or by in-directly by production of siderophores, ammonia, $\mathrm{HCN}$, enzymes and secondary metabolites. ${ }^{16,17}$ The plant growth promoting methylotrophs as single bioinoculants or with co-inoculated as microbial consortium may be use as bioinoculants/biofertilizers of biocontrol agents for enhanced crops production and soil fertility for sustainable agriculture. ${ }^{18-21}$

The different class $\alpha, \beta$ and $\gamma$-proteobacteria of methylotrophic bacteria communities have been reported worldwide. The class $\alpha$-proteobacteria has been reported as most dominant followed by $\beta$-proteobacteria. The novel methylotrophic microbes have been isolated and characterized from different habitats worldwide including Methylocella silvestris BL2T , Methylocella palustris KT, Methyloferula stellata AR4T and Methylocapsa acidiphila B2T from acidic soil:;2-25 Methylobacterium tarhaniae N4211T from arid soil;:26 Methylobacterium iners 5317S-33T and Methylobacterium aerolatum 5413S-11T from air sample; ${ }^{27}$ Methylobacterium adhaesivum AR27T and Methylobacterium isbiliense AR24T from drinking water, ${ }^{28,29}$ Methylobacterium brachiatum B0021T, Methylobacterium gregans 002-074T, Methylobacterium komagatae 002-079T, Methylobacterium persicinum 002-165T and Methylobacterium tardum RB677T from freshwater sample; ${ }^{30}$ Methylobacterium organophilum XX, Methylotenera versatilis 301T and Methylotenera mobilis JLW8T from lakes; ${ }^{31-33}$ Methylobacterium brachythecii $99 \mathrm{bT}$, Methylobacterium cerastii C44, Methylobacterium gnaphalii 23eT, Methylobacterium gossipiicola Gh-105T, Methylobacterium haplocladii 87eT, Methylobacterium oxalidis 35aT, Methylobacterium phyllosphaerae B27T, Methylobacterium phyllostachyos BL47T, Methylobacterium platani PMB02T, Methylobacterium pseudosasicola BL36T, Methylobacterium thuringiense C34T, Methylobacterium trifolii TA73T, from leaf surface of diverse plants; $;^{34-42}$ Methylobacterium aminovorans TH-1, Methylobacterium goesingense iEII3, Methylobacterium soli YIM 48816T, Methylobacterium suomiense, F20T, Methylobacterium thiocyanatum, Methylobacterium variabile GR3T, Methylopila capsulata IM1T, and Methylopila helvetica VKMB-189 from soil samples ${ }^{43-50}$

To understand the mechanisms of plant growth promotion and genes involved in plant growth promotion there are many reports on
Volume 5 Issue 6 - 2018

\author{
Neelam Yadav,' Ajar Nath Yadav ${ }^{2}$ \\ 'Gopi Nath PG College,Veer Bahadur Singh Purvanchal \\ University, India \\ 2Department of Biotechnology, Akal College of Agriculture, \\ Eternal University, India
}

Correspondence: Ajar Nath Yadav, Department of Biotechnology, Akal College of Agriculture, Eternal University, Baru Sahib, India,Tel +9I-9882545085, Fax: +9| I799276006, Email ajavbiotech@gmail.com

Received: November 07, 2018 | Published: November 20, 2018

whole genome sequences of methylotrophic bacteria are available at NCBI GenBank database (https://www.ncbi.nlm.nih.gov) Methylobacterium populi BJ001, Methylobacterium extorquens CM4, Methylobacterium nodulans ORS 2060, Methylobacterium aquaticum MA-22A, Methylobacterium radiotolerans JCM 2831, Methyloferula stellata AR4, Methylotenera mobilis JLW8, Methylotenera versatilis 301, Methylobacterium sp. AMS5, Methylotenera versatilis 301 Methylotenera mobilis JLW8, Methylovorus glucosetrophus SIP3-4, Methylovorus glucosetrophus SIP3-4, Methylobacterium mesophilicum SR1.6/6, and Methylobacterium indicum SE2.11

Plant-associated methylotrophs produce PGP phytohormones such as auxins, gibberellins and cytokinin by Methylobacterium extorquens IIWP-43, M. extorquens MP1, M. mesophilicum B-2143, M. mesophilicum HHS1-36, M. mesophilicum IIWP-45, M. mesophilicum NIAW1-41, M. phyllosphaerae HHS2-67, M. radiotolerans HHS1-45, $M$. radiotolerans IHD-35 and M. zatmanii MS4. Many methylotrophs has been reported to fix N2 e.g. Methylobacterium mesophilicum B-2143, M. nodulans 2060T, and Methylobacterium sp. THD-3511, ${ }^{51-}$ ${ }^{56}$. A vast number methylotrophs with P-solubilizing ability have been reported Methylobacillus arboreus Iva, M. extorquens G10, M. extorquens IIWP-43, M. lusitanum MSF 32, M. mesophilicum IIWP-45, M. mesophilicum NIAW1-41, M. radiotolerans IHD-35, Methylopila musalis MUSA and Methylovorus menthalis MM. 2,6,13,57,58

\section{Conclusion}

The methylotrophic microbes from diverse sources have potential applications in agriculture, industry and allied sectors. The methylotrophic bacteria could be used for plant growth and soil health for sustainable agriculture when inoculated as single or as consortium under the natural as well as abiotic stress conditions.

\section{Acknowledgements}

The authors are grateful to Prof. Harcharan Singh Dhaliwal, Vice Chancellor, Eternal University, Baru Sahib, Himachal Pradesh, India for providing infra-structural facilities and constant encouragement. 


\section{Conflict of interest}

All authors declare that they have no conflicts of interest to this work.

\section{References}

1. Röling W, Ortega-Lucach S, Larter S, et al. Acidophilic microbia communities associated with a natural, biodegraded hydrocarbon seepage. J Appl Microbiol. 2006;101(2):290-299.

2. Verma P, Yadav AN, Kazy SK, et al. Elucidating the diversity and plant growth promoting attributes of wheat (Triticum aestivum) associated acidotolerant bacteria from southern hills zone of India. Natl J Life Sci. 2013;10:219-227.

3. Trotsenko YA, Doronina N, Li TD, et al. Moderately haloalkaliphilic aerobic methylobacteria. Microbiology. 2007;76:253-265.

4. Doronina NV, Poroshina MN, Kaparullina EN, et al. Methyloligella halotolerans gen. nov., sp. nov. and Methyloligella solikamskensis sp. nov, two non-pigmented halotolerant obligately methylotrophic bacteria isolated from the Ural saline environments. Syst Appl Microbiol. 2013;36:148-154.

5. Shmareva M, Doronina N, Tarlachkov S, et al. Methylophaga muralis Bur 1, a haloalkaliphilic methylotroph isolated from the Khilganta soda lake (Southern Transbaikalia, Buryat Republic). Microbiology. 2018;87:3346

6. Verma P, Yadav AN, Kazy SK, et al. Evaluating the diversity and phylogeny of plant growth promoting bacteria associated with wheat (Triticum aestivum) growing in central zone of India. Int J Curr Microbiol Appl Sci. 2014;3:432-447.

7. Sivakumar R, Nandhitha G, Chandrasekaran P, et al. Impact of Pink Pigmented Facultative Methylotroph and PGRs on Water Status, Photosynthesis, Proline and NR Activity in Tomato under Drought. Int J Curr Microbiol App Sci. 2017;6:1640-1651.

8. Kerry RG, Patra S, Gouda S, et al. Microbes and Their Role in Drought Tolerance of Agricultural Food Crops. In: Microbial Biotechnology. Springer. 2018;1:253-273.

9. Romanovskaia V, Shilin S, Chernaia N, et al. Search for psychrophilic methylotrophic bacteria in biotopes of the Antarctica. Mikrobiol. Z 2005;67:3-8.

10. Yadav AN. Bacterial diversity of cold deserts and mining of genes for low temperature tolerance. Ph.D. thesis, IARI, New Delhi/BIT, Ranchi; 2015. 234 p.

11. Verma P, Yadav AN, Khannam KS, et al. Assessment of genetic diversity and plant growth promoting attributes of psychrotolerant bacteria allied with wheat (Triticum aestivum) from the northern hills zone of India. Ann Microbiol. 2015;65:1885-1899.

12. Amin A, Ahmed I, Salam N, et al. Diversity and distribution of thermophilic bacteria in hot springs of Pakistan. Microb Ecol. 2017;74:116-127.

13. Verma P, Yadav AN, Khannam KS, et al. Appraisal of diversity and functional attributes of thermotolerant wheat associated bacteria from the peninsular zone of India. Saudi J Biol Sci. 2016.

14. Yadav AN. Studies of Methylotrophic Community from the Phyllosphere and Rhizosphere of Tropical Crop Plants, M.Sc. Thesis, Bundelkhand University; 2009. 66 p.

15. Lacava PT, Silva-Stenico ME, Araújo WL, et al. Detection of siderophores in endophytic bacteria Methylobacterium spp. associated with Xylella fastidiosa subsp pauca. Pesq Agro Brasil. 2008;43:521-528.
16. Madhaiyan M, Suresh Reddy BV, Anandham R et al. Plant GrowthPromoting Methylobacterium Induces Defense Responses in Groundnut (Arachis hypogaea L.) Compared with Rot Pathogens. Curr Microbiol. 2006;53:270-276.

17. Madhaiyan M, Poonguzhali S, Senthilkumar M et al. Growth promotion and induction of systemic resistance in rice cultivar Co-47 (Oryza sativa L.) by Methylobacterium spp. Bot Bull Acad Sin. 2004. 45 p.

18. Yadav AN, Verma P, Kour D, et al. Plant microbiomes and its beneficial multifunctional plant growth promoting attributes. Int $J$ Environ Sci Nat Resour. 2017;3:1-8.

19. Yadav AN, Verma P, Kumar R, et al. Current applications and future prospects of eco-friendly microbes. EU Voice. 2017;3:21-22

20. Yadav AN, Kumar R, Kumar S et al. Beneficial microbiomes: Biodiversity and potential biotechnological applications for sustainable agriculture and human health. J Appl Biol Biotech. 2017;5:1-13.

21. Verma P, Yadav AN, Khannam KS, et al. Molecular diversity and multifarious plant growth promoting attributes of Bacilli associated with wheat (Triticum aestivum $\mathrm{L}$.) rhizosphere from six diverse agro-ecological zones of India. J Basic Microbiol. 2016;56:44-58.

22. Dunfield PF, Khmelenina VN, Suzina NE, et al. Methylocella silvestris sp. nov., a novel methanotroph isolated from an acidic forest cambisol. Int $J$ Syst Evol Microbiol. 2003;53:1231-1239.

23. Dedysh SN, Liesack W, Khmelenina VN, et al. Methylocella palustris gen. nov., sp. nov., a new methane-oxidizing acidophilic bacterium from peat bogs, representing a novel subtype of serine-pathway methanotrophs. Int J Syst Evol Microbiol. 2000;50:955-969.

24. Vorobev AV, Baani M, Doronina NV, et al. Methyloferula stellata gen. nov., sp. nov., an acidophilic, obligately methanotrophic bacterium that possesses only a soluble methane monooxygenase. Int $J$ Syst Evol Microbiol. 2011;61:2456-2463.

25. Dedysh SN, Khmelenina VN, Suzina NE, et al. Methylocapsa acidiphila gen. nov., sp. nov., a novel methane-oxidizing and dinitrogen-fixing acidophilic bacterium from Sphagnum bog. Int J Syst Evol Microbiol. 2002;52:251-261.

26. Veyisoglu A, Camas M, Tatar D, et al. Methylobacterium tarhaniae sp. nov., isolated from arid soil. Int J Syst Evol Microbiol. 2013;63:28232828.

27. Weon H-Y, Kim B-Y, Joa JH, et al. Methylobacterium iners sp. nov. and Methylobacterium aerolatum sp. nov., isolated from air samples in Korea. Int J Syst Evol Microbiol. 2008;58:93-96.

28. Gallego V, García MT, Ventosa A. Methylobacterium adhaesivum sp. nov., a methylotrophic bacterium isolated from drinking water. Int J Syst Evol Microbiol. 2006;56:339-342.

29. Gallego V, García MT, Ventosa A. Methylobacterium isbiliense sp. nov. isolated from the drinking water system of Sevilla, Spain. Int J Syst Evol Microbiol. 2005;55:2333-2337.

30. Kato Y, Asahara M, Goto K, et al. Methylobacterium persicinum sp. nov., Methylobacterium komagatae sp. nov., Methylobacterium brachiatum sp. nov., Methylobacterium tardum sp. nov. and Methylobacterium gregans sp. nov., isolated from freshwater. Int J Syst Evol Microbiol. 2008;58:1134-1141

31. Patt TE, Cole GC, Hanson RS. Methylobacterium, a New Genus of Facultatively Methylotrophic Bacteria. Int J Syst Evol Microbiol. 1976;26:226-229.

32. Kalyuzhnaya MG, Beck DAC, Vorobev A et al. Novel methylotrophic isolates from lake sediment, description of Methylotenera versatilis $\mathrm{sp}$. 
nov. and emended description of the genus Methylotenera. Int J Syst Evol Microbiol. 2012;62:106-111.

33. Kalyuzhnaya MG, Bowerman S, Lara JC, et al. Methylotenera mobilis gen. nov., sp. nov., an obligately methylamine-utilizing bacterium within the family Methylophilaceae. Int J Syst Evol Microbiol. 2006;56:28192823.

34. Tani A, Sahin N, Kimbara K. Methylobacterium oxalidis sp. nov., isolated from leaves of Oxalis corniculata. Int J Syst Evol Microbiol. 2012;62:1647-1652.

35. Kang YS, Kim J, Shin HD, et al. Methylobacterium platani sp. nov., isolated from a leaf of the tree Platanus orientalis. Int J Syst Evol Microbiol. 2007;57:2849-2853.

36. Wellner S, Lodders N, Glaeser SP, et al. Methylobacterium trifolii sp. nov. and Methylobacterium thuringiense sp. nov., methanol-utilizing, pinkpigmented bacteria isolated from leaf surfaces. Int J Syst Evol Microbiol. 2013;63:2690-2699.

37. Wellner S, Lodders N, Kämpfer P. Methylobacterium cerastii sp. nov., isolated from the leaf surface of Cerastium holosteoides. Int J Syst Evol Microbiol. 2012;62:917-924.

38. Madhaiyan M, Poonguzhali S, Kwon SW, et al. Methylobacterium phyllosphaerae sp. nov., a pink-pigmented, facultative methylotroph from the phyllosphere of rice. Int J Syst Evol Microbiol. 2009;59:22-27.

39. Madhaiyan M, Poonguzhali S. Methylobacterium pseudosasicola sp. nov. and Methylobacterium phyllostachyos sp. nov., isolated from bamboo leaf surfaces. Int J Syst Evol Microbiol. 2014;64:2376-2384.

40. Tani A, Sahin N. Methylobacterium haplocladii sp. nov. and Methylobacterium brachythecii sp. nov., isolated from bryophytes. Int $J$ Syst Evol Microbiol. 2013;63:3287-3292.

41. Madhaiyan M, Poonguzhali S, Senthilkumar M, et al. Methylobacterium gossipiicola sp. nov., a pink-pigmented, facultatively methylotrophic bacterium isolated from the cotton phyllosphere. Int J Syst Evol Microbiol. 2012;62:162-167.

42. Tani A, Sahin N, Kimbara K. Methylobacterium gnaphalii sp. nov., isolated from leaves of Gnaphalium spicatum. Int J Syst Evol Microbiol. 2012;62:2602-2607.

43. Cao YR, Wang Q, Jin RX, et al. Methylobacterium soli sp. nov. a methanol-utilizing bacterium isolated from the forest soil. Antonie van Leeuwenhoek. 2011;99:629-634.

44. Idris R, Kuffner M, Bodrossy L, et al. Characterization of Ni-tolerant methylobacteria associated with the hyperaccumulating plant Thlaspi goesingense and description of Methylobacterium goesingense sp. nov. Syst Appl Microbiol. 2006;29:634-644.

45. Gallego V, García MT, Ventosa A. Methylobacterium variabile sp. nov., a methylotrophic bacterium isolated from an aquatic environment. Int J Syst Evol Microbiol. 2005;55:1429-1433.
46. Doronina NV, Trotsenko YA, Kuznetsov BB, et al. Methylobacterium suomiense sp. nov. and Methylobacterium lusitanum sp. nov., aerobic, pink-pigmented, facultatively methylotrophic bacteria. Int J Syst Evol Microbiol. 2002;52:773-776.

47. Doronina NV, Trotsenko YA, Tourova TP, et al. Methylopila helvetica sp. nov. and Methylobacterium dichloromethanicum sp. nov. - Novel Aerobic Facultatively Methylotrophic Bacteria Utilizing Dichloromethane. Syst Appl Microbiol. 2000;23:210-218.

48. Wood AP, Kelly DP, McDonald IR et al. A novel pink-pigmented facultative methylotroph, Methylobacterium thiocyanatum sp. nov., capable of growth on thiocyanate or cyanate as sole nitrogen sources. Arch Microbiol. 1998;169:148-158.

49. Doronina NV, Trotsenko YA, Krausova VI, et al. Methylopila capsulata gen. nov., sp. nov., a novel non-pigmented aerobic facultatively Methylotrophic bacterium. Int J Syst Evol Microbiol. 1998;48:1313-1321.

50. Urakami T, Araki H, Suzuki KI, et al. Further Studies of the Genus Methylobacterium and Description of Methylobacterium aminovorans sp. nov. Int J Syst Evol Microbiol. 1993;43:504-513.

51. Ivanova E, Doronina N, Trotsenko YA. Aerobic Methylobacteria are capable of synthesizing auxins. Microbiology. 2001;70:392-397.

52. Trotsenko YA, Ivanova E, Doronina N. Aerobic methylotrophic bacteria as phytosymbionts. Microbiology. 2001;70:623-632.

53. Omer Z, Tombolini R, Broberg A, et al. Indole-3-acetic acid production by pink-pigmented facultative Methylotrophic bacteria. Plant Growth Reg. 2004;43:93-96.

54. Pattnaik S, Rajkumari J, Paramanandham P, et al. Indole Acetic Acid Production and Growth-Promoting Activity of Methylobacterium extorquens MP1 and Methylobacterium zatmanii MS4 in Tomato. Int J Veg Sci. 2017;23:321-330.

55. Chanratana M, Han GH, Roy Choudhury A, et al. Assessment of Methylobacterium oryzae CBMB20 aggregates for salt tolerance and plant growth promoting characteristics for bio-inoculant development. AMB Exp. 2017;7:208.

56. Meena KK, Kumar M, Kalyuzhnaya MG, et al. Epiphytic pink-pigmented Methylotrophic bacteria enhance germination and seedling growth of wheat (Triticum aestivum) by producing phytohormone. Antonie van Leeuwenhoek. 2012;101(4):777-786.

57. Agafonova N, Kaparullina E, Doronina N, et al. Phosphate-solubilizing activity of aerobic methylobacteria. Microbiology. 2013;82:864-867.

58. Jayashree S, Vadivukkarasi P, Anand K, et al. Evaluation of pink-pigmented facultative Methylotrophic bacteria for phosphate solubilization. Arch Microbiol. 2011;193:543-552. 